\title{
COLLISION TUMOR OF OVARY ASSOCIATED WITH CONTRALATERAL BENIGN CYSTIC TERATOMA IN PRIMIGRAVIDA: A CASE TO REMEMBER
}

Thakur Brijesh¹, Kishore Sanjeev², Bhardwaj Aparna ${ }^{3}$, Kudesia Sandip ${ }^{4}$ Neelima Bahal $^{5}$

\section{HOW TO CITE THIS ARTICLE:}

Thakur Brijesh, Kishore Sanjeev, Bhardwaj Aparna, Kudesia Sandip, Neelima Bahal "Collision Tumor of Ovary Associated with Contralateral Benign Cystic Teratoma in Primigravida: A Case to Remember". Journal of Evolution of Medical and Dental Sciences 2014; Vol. 3, Issue 36, August 18; Page: 9489-9493,

DOI: $10.14260 /$ jemds/2014/3225

ABSTRACT: Collision tumors involving ovaries are extremely rare. Here, we report this rare entity in a 23 years old primigravida presented with 12 weeks pregnancy and abdominal pain. Ultrasonography showed large right adnexal mass measuring $20 \times 13 \mathrm{~cm}$. having solid area with multiple cysts and bulky left ovary of $6 \times 4.6 \mathrm{~cm}$. Normal ovarian tissue was preserved after bilateral ovarian cystectomy. Grossly, right ovarian tissue revealed multiloculation and mucin filled cysts. Left ovarian cyst was filled with hairy pultaceous material. Histological diagnosis of collision tumor of right ovary comprising hemorrhagic mucinous cystadenoma and benign cystic teratoma along with dermoid cyst in left ovary was rendered. The pathogenesis of association of mature cystic teratoma with a mucinous cystadenoma has yet to be answered and the choice of management necessitates a weighing of risks based on characterization of the adnexal mass and gestational age.

KEYWORDS: Collision, mucinous, ovary, primigravida, pregnancy.

INTRODUCTION: Collision tumor means the coexistence of two adjacent, but histologically distinct tumors without histologic admixture in the same tissue or organ. [1] Either two different types of tumors may arise independently or metachronously at the same place or one or both malignancies may metastasize to the same location. They must be differentiated from "composite" tumors, which consist of a malignant neoplasm that has differentiated into two distinct subtypes. [2]

Collision tumors have been reported at various sites many of which include skin, gastrointestinal tract, thyroid and ovaries. $[2,3,4]$ Collision tumors involving ovaries are extremely rare. Most common histologic combination of collision tumor in the ovary is coexistence of teratoma with mucinous tumors. $[1,5]$

Here, to the best of our knowledge, we are describing the first case of collision tumor of right ovary comprising benign cystic teratoma and mucinous cystadenoma coexisting with contralateral benign cystic teratoma presenting in first trimester in primigravida.

CASE REPORT: A 23 years old married female presented with amenorrhea of 12 weeks and complained of abdominal pain for last 5 days. Hematological investigations revealed $\mathrm{Hb} 8.1 \mathrm{gm} \%$ and mild leukocytosis (TLC $=12700$ cells $/ \mathrm{mm}^{3}$ ). Liver and renal function test were within normal limit. Serum alpha fetoprotein $(6.9 \mathrm{IU} / \mathrm{ml})$ and CA-125 $(73.7 \mathrm{U} / \mathrm{ml})$ levels were increased.

Serum LDH level was found to be normal (475 U/L). Ultrasonography showed large right adnexal complex mass measuring approx. $20 \times 13 \mathrm{~cm}$. having solid area with nearly half of mass occupied by multiple cysts. Left ovary was also bulky measuring $6 \times 4.6 \mathrm{~cm}$. and seen in pouch of Douglas. USG revealed an intrauterine fetal pole measuring $5.6 \mathrm{~cm}$. corresponding to 12 weeks 1 day with regular fetal heart rate (fig. 1.a). 
Laparotomy with bilateral cystectomy was planned. Peroperatively, uterus was enlarged upto 14 weeks of size with slight blood stained fluid in pouch of Douglas. Left sided ovary measuring 8 x 8 $\mathrm{cm}$. was found to be dermoid cyst grossly. A large right sided ovarian tumor of $30 \mathrm{x} 28 \mathrm{~cm}$. in size with omental adhesions on superior surface was found to be twisted twice on its pedicle.

Peroperative frozen sections were reported as suggestive of benign cystadenoma (fig. 1.b). Normal ovarian tissue was preserved after bilateral ovarian cystectomy. Peritoneal fluid sent for cytological examination showed hemorrhagic background, sheets of mesothelial cells with a few clusters of cells exhibiting mild to moderate atypia and was reported positive for atypical cells.

Grossly, excised right ovarian tissue was received as two dark brown irregular pieces measuring $12.5 \times 10 \mathrm{~cm}$. and $13 \times 7.5 \mathrm{~cm}$. exhibiting multiloculation and variable sized mucin filled cysts (fig. 1c). Microscopy showed a combination of morphological features. Multiple sections from right ovarian tissue revealed large amount of blood and fibrin clots with fibrous cyst wall lined by mucin secreting tall columnar cells at places and a small keratin filled cyst lined by stratified squamous epithelium along with focal collection of mature adipocytes (fig. 2a, b, c).

None of the sections showed histological evidence of direct transition from one tumor to other. Left ovarian tissue showed already cut open cystic mass measuring $3.5 \times 3.0 \mathrm{~cm}$. which was filled with pultaceous material and hairs. Left ovarian tissue showed mature fat, cartilage, skin epithelium with pilosebaceous units and sweat glands (fig. 2d). Histological diagnosis of hemorrhagic mucinous cystadenoma with small benign cystic teratoma in right ovary and benign cystic teratoma in left ovary was considered. Postoperative period of two months was uneventful.

DISCUSSION: The definition for a collision tumor is the concrescence of two neighboring independent neoplasms. When two types of tissue are intermingled within the same tumor, the term "composite" is used, while when they are adjacent to one another without intermixing or separated by narrow stroma or their respective basal lamina, the term "collision" is generally accepted.[1,4] Various hypothesis for the formation of collision tumor have been given.

Accidental presence of two primary tumors, common stem cell of origin, common carcinogenic agent or one tumor providing microenvironment for the growth of other tumor may be some of the possibilities. [3] Incidence of collision tumors in ovary is very rare and even rarer in pregnancy. ${ }^{[6]}$ There are instances of ovarian teratomas coexisting with serous, mucinous or granulose cell tumors in the available literature. [7]

Benign cystic teratomas of the ovary are among the common benign neoplasms of the ovary commonly seen occurring in young patients mostly diagnosed between 20 and 30 years of age. [8]

Mucinous tumors are multiloculated cysts lined by epithelium resembling that of the endocervix. Teratomas reveal tissues originating from the ectoderm, mesoderm and endoderm while Mucinous cystadenomas have a controversial histogenesis.

Some proposals suggest their origin from metaplasia of surface epithelium or a teratomatous origin. [9] But the relationship between the pathogenesis of the two lesions occurring in same or contralateral ovary has not yet been answered.

Widespread use of ultrasound in early pregnancy has led to the detection of incidental adnexal masses more frequently. Characterization of an ovarian mass with complexity, septations, solid component nodules, papillary components and size can assist with the prediction of malignancy. 
An adnexal mass in pregnancy can be complicated by torsion, rupture, or bleeding/infection, or labor obstruction.

Management can be conservative or surgical depending on the size, gestational age, available resources, and possibly patient preference following careful evaluation. For those masses suspicious of malignancy, at risk of torsion, rupture, or clinically symptomatic, surgical treatment is warranted. [10]

This case report was considered worth documentation since this triple coexistence of bilateral benign cystic teratoma with one sided large mucinous cystadenoma in pregnancy is a rarest entity and first case report as far as we know. The possibility of a collision tumor should be considered when an ovarian teratoma has imaging findings that cannot be explained solely by an ovarian teratoma.

The possible existence of an ovarian collision tumor should carefully be examined pre- and postoperatively and histologically, so as to avoid misdiagnosis of a possible malignancy. CA125 is typically elevated during the first trimester, but may be useful during later assessment or follow-up. So, tumor markers should be used with caution, particularly in the first trimester, because of the wide variation in results and interpretation.

Sometimes rupture of cystic ovarian mass or spillage of cystic fluid may cause chemical or granulomatous peritonitis. These changes can mimic malignancy and can be misdiagnosed. Malignancy risk should be discarded in bilateral ovarian cysts with large size, intra-cystic hemorrhage and semisolid mass.

The reporting of similar cases with longer follow up will shed more light on the behavior of collision tumors. They should be classified in appropriate manner so that we can point out clearly enough the dualistic nature of collision tumors. Moreover they can be confusing for clinicians and do not reflect their needs in terms of leading to the correct therapeutic options.

In conclusion, collision tumors should be extensively examined to avoid misdiagnosis and the choice of management necessitates a weighing of risks based on characterization of the adnexal mass and gestational age. Such cases should be highlighted for academic as well as patient's diagnosis, optimal therapy and prognosis.

\section{REFERENCES:}

1. Bige O, Demir A, Koyuncuoglu M, Secil M, Ulukus C, Saygili U. Collision tumor: serous cystadenocarcinoma and dermoid cyst in the same ovary. Archives of Gynecology and Obstetrics. 2009; 279 (5): 767-770.

2. Coupland SE, Dodson A, Liu H, Du MQ, Angi M, Damato BE. Intraocular collision tumour: case report and literature review. Graefe's Archive for Clinical and Experimental Ophthalmology. 2013; 251 (5): 1383-1388.

3. Milhan ABM, Sheriff AMM, Jayawikrama MMA. Collision tumour; Papillary thyroid cancer colliding with squamous oesophageal cancer. Ceylon Journal of Otolaryngology. 2013; 3 (1): 27-28.

4. Mrozi A, Kiedrowski M, Malinowska M, Sopylo R. Collision Tumor of the stomach Adenocarcinoma and Neuroendocrine carcinoma: Case Report and Review of the Literature. Pol J Pathol. 2009; 2: 94-97. 
5. Lee NH, Hong JH, Kim YS, Mun ST, Jeon S, Sunwoo JG et al. A case of laparoscopic treatment of collision tumor: Huge serous cystadenoma and dermoid cyst arising in the same ovary. Korean J Obstet Gynecol. 2010; 53 (1): 90-94.

6. Papaziogas B, Souparis A, Grigoriou M, Tsiaousis P, Kogia E, Panagiotopoulou K et al. A Rare Triple Coexistence of a Collision Tumor, a Benign Mature Cystic Teratoma and a Hemorrhagic Follicular Cyst of the Ovaries. The Internet Journal of Surgery. 2007; 14 (1).

7. Choudhary S, Adisesha S. Collision tumors of ovary: A rare phenomenon. International Journal of Case Reports and Images. 2012; 3 (10): 68-70.

8. Sachchidananda Maiti S, Zamurrad Fatima Z, Anjum ZK, Hopkins RE. Ruptured ovarian cystic teratoma in pregnancy with diffuse peritoneal reaction mimicking advanced ovarian malignancy: a case Report. Journal of Medical Case Reports. 2008; 2:203

9. Parveen S, Hakim S, Fatima Z. Mature Cystic Teratoma Of The Ovary Associated With Contralateral Mucinous Cystadenoma: a Case Report. Indian Journal of Applied Research. 2012; 1 (12): 176-177.

10. Yakasai IA, Bappa LA. Diagnosis and management of adnexal masses in pregnancy. J Surg Tech Case Rep. 2012; 4 (2): 79-85.

\section{Figure: 1.}

a) Preoperative USG showing large multiloculated cystic ovarian mass with intrauterine gestational sac.

b) Peroperative frozen section showing cyst lined by mucin secreting tall columnar epithelium (H \& E X40). Inset showing higher magnification (H \& E X400).

c) Gross specimen of excised right ovarian tissue exhibiting multiloculation and variable sized mucin filled cysts.

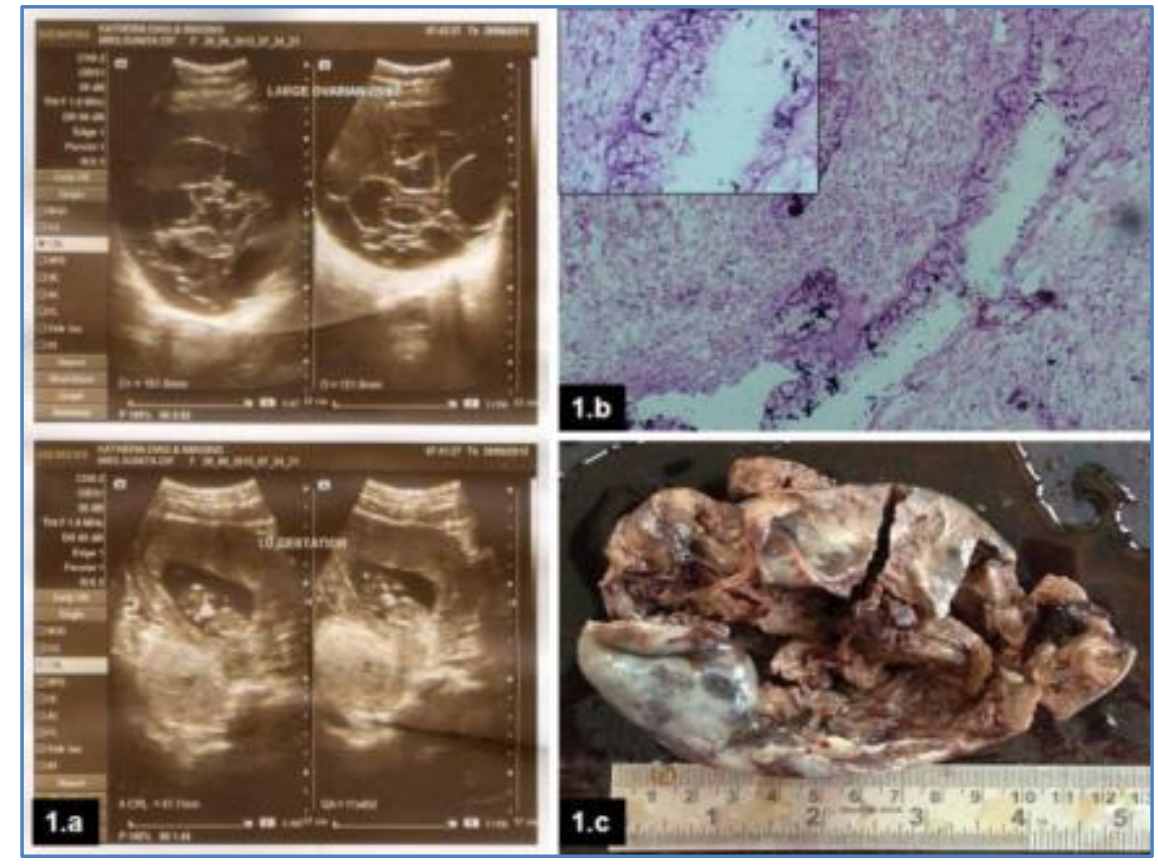

Fig. 1a, 1b, 1c 
Figure: 2.

a. Right ovarian tissue showing cyst lined by stratified squamous epithelium with lamellated keratin (H \& E X100). Inset showing higher magnification (H \& E X400).

b. Focal collection of mature adipocytes in right ovarian tissue (H \& E X100).

c. Right ovarian cyst lined by mucin secreting tall columnar epithelium with dilated, congested blood vessels and areas of haemorrhage in the cyst wall (H \& E X40). Inset showing higher magnification (H \& E X400).

d. Left ovary showing mature cartilage (H \& E X40). Inset showing adnexal glands (H \& E X400).

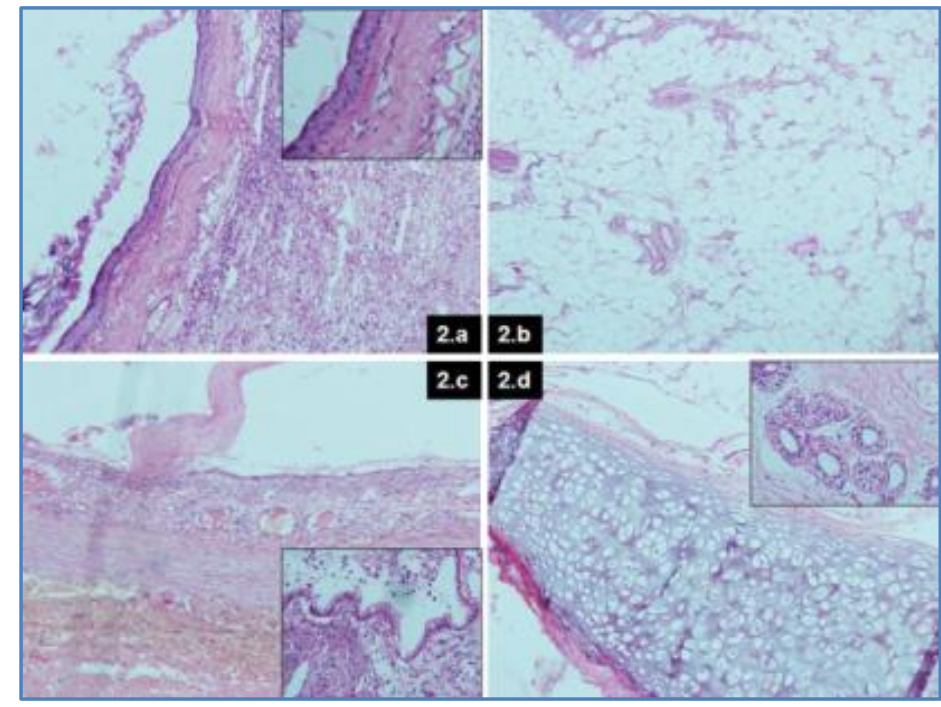

Fig. 2a, 2b, 2c, 2d

\section{AUTHORS:}

1. Thakur Brijesh

2. Kishore Sanjeev

3. Bhardwaj Aparna

4. Kudesia Sandip

5. Neelima Bahal

\section{PARTICULARS OF CONTRIBUTORS:}

1. Assistant Professor, Department of Pathology, SGRRIM \& HS, Dehradun.

2. Professor, Department of Pathology, SGRRIM \& HS, Dehradun.

3. Associate Professor, Department of Pathology, SGRRIM \& HS, Dehradun.

4. Professor and HOD, Department of Pathology, SGRRIM \& HS, Dehradun.
5. Junior Resident, Dept of Pathology, SGRRIM\&HS, Dehradun.

\section{NAME ADDRESS EMAIL ID OF THE CORRESPONDING AUTHOR:}

Dr. Brijesh Thakur, Department of Pathology, SGRRIM \& HS, Dehradun, Uttrakhand, India.

Email: drbrijeshthakur03@gmail.com

Date of Submission: 18/07/2014.

Date of Peer Review: 19/07/2014.

Date of Acceptance: 11/08/2014.

Date of Publishing: 18/08/2014. 